\title{
Origine et développement des bactéries coliformes dans les fromages à pâte molle*
}

\author{
par \\ R. MOURGUES, L. VASSAL, J. AUCLAIR, G. MOCQUOT \\ Station Centrale de Recherches Laitières, I.N.R.A. \\ 78350 Jouy-en-Josas (France) \\ et J. VANDEWEGHE \\ Union des Coopératives Agricoles Laitières de l'Yonne \\ et de la Nièvre - 89470 Monéteau (France) \\ avec la collaboration technique de N. Deschamps, J.C. Vachot \\ et M. NICOLAS
}

\section{INTRODUCTION}

Les fromages à pâte molle fabriqués à partir de lait pasteurisé sont fréquemment contaminés en cours de fabrication par des microorganismes dont le développement pendant la fabrication même, puis au cours de l'affinage et de la conservation des fromages au froid, peut présenter un risque au point de vue de l'hygiène. C'est en particulier le cas des germes appartenant au groupe des coliformes que l'on rencontre très souvent dans les fromages à pâte molle, quelquefois en nombres élevés. Si beaucoup de ces micro-organismes sont considérés comme non pathogènes, certains d'entre eux, notamment ceux appartenant à l'espèce Escherichia coli, sont considérés par les épidémiologistes comme éventuellement responsables d'intoxications alimentaires.

C'est ainsi qu'en novembre 1971 aux Etats-Unis une intoxication alimentaire survenue à la suite de l'ingestion de fromages à pâte molle importés de France a été attribuée à la présence de $10^{5}$ à $10^{7}$ $E$. coli entérotoxiques par gramme dans les fromages incriminés $[1,2]$. Cette intoxication a, par la suite, amené la « Food and Drug Administration " à contrôler de près la qualité hygiénique des fromages à pâte molle importés.

\footnotetext{
* Ce travail a bénéficié d'une subvention de la Délégation Générale à la Recherche Scientifique et Technique (contrat $\mathrm{n}^{\circ}$ 73.7.1534).
} 
Il est donc très utile de savoir si la présence de nombres élevés de bactéries coliformes dans les fromages à pâte molle prêts à la consommation provient :

a) d'une contamination au stade du lait et de la fabrication,

b) d'un processus technologique de nature à favoriser le développement des coliformes, même si ces derniers sont peu nombreux dans le lait ou

c) d'un développement excessif de ces micro-organismes au cours de la fabrication, de l'affinage ou de la conservation au froid des fromages.

L'objectif du présent travail était de déterminer sur un nombre suffisant de fabrications fromagères l'origine des contaminations du fromage en bactéries coliformes, le rôle du processus technologique dans le développement de cette flore microbienne et les conditions de survie ou de développement des bactéries coliformes au cours de l'affinage, de la conservation, du transport et de la commercialisation des fromages.

\section{METHODES ET RESULTATS}

I.-Dans une PREMIERE SERIE D'ESSAIS de fabrication de fromages de type Camembert, conduite à la Laiterie Expérimentale de Jouy-enJosas, on a suivi les variations du nombre des bactéries coliformes présentes dans le lait de fabrication, dans le caillé au cours de la fabrication et dans les fromages au cours de leur affinage et de leur conservation au froid. Le but ultime de ces essais était de déterminer les conditions de fabrication qui permettent d'obtenir des fromages affinés exempts de bactéries coliformes.

\section{Méthodes}

\section{1) FABRiCATION ET AFFINAGE DU FROMAGE}

Seize essais ont ainsi été réalisés, suivant le procédé de fabrication traditionnel, en utilisant chaque fois 1001 de lait pasteurisé (chauffage à $72^{\circ} \mathrm{C}$ pendant $15 \mathrm{~s}$ ). La figure 1 représente l'évolution de la température dans le lait, le caillé et le fromage au cours de la fabrication et de l'affinage.

Au cours des huit premiers essais on faisait subir au lait une " maturation » qui consistait, après la pasteurisation et le refroidissement du lait à $12^{\circ} \mathrm{C}-15^{\circ} \mathrm{C}$, à ensemencer ce lait avec des ferments lactiques (inoculum 0,015 p. 100 d'une suspension concentrée congelée de streptocoques lactiques, correspondant à 1,5 p. 100 d'un levain sur lait classique et à un nombre initial de bactéries lactiques voisin de $1,5.10^{7}$ par $\mathrm{ml}$ de lait inoculé). Le lait était maintenu à la même température $\left(12^{\circ} \mathrm{C}\right.$ à $\left.15^{\circ} \mathrm{C}\right)$ jusqu'à l'emprésurage, c'est-à-dire pendant environ $17 \mathrm{~h}$. Dans ces conditions la flore lactique se 


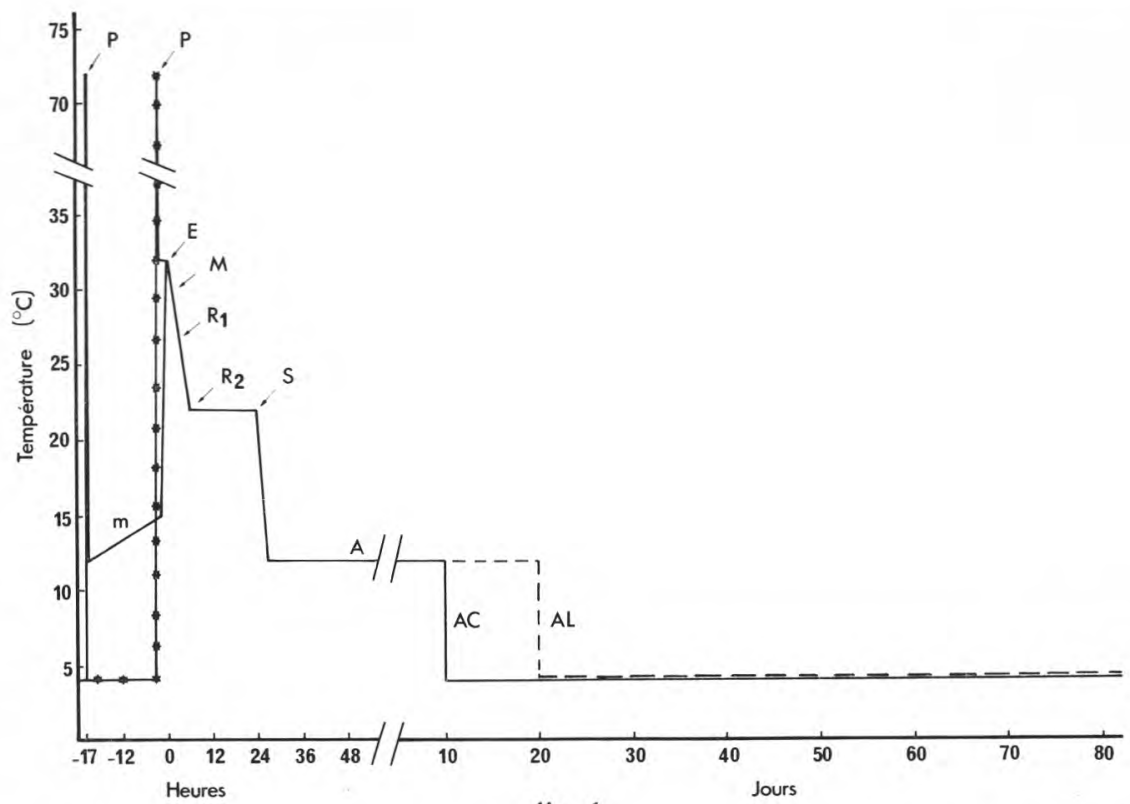

fig. 1

Evolution de la température dans le lait, le caillé et le fromage au cours de la fabrication et l'affinage des fromages expérimentaux.
$\mathbf{P}=$ pasteurisation,
$\mathrm{m}$ = maturation,
$\mathrm{E}=$ emprésurage,
$\mathbf{M}=$ mise en moules,
$\mathrm{R} 1=1^{\mathrm{er}}$ retournement,
$\mathrm{R} 2=2^{\mathrm{e}}$ retournement,
$\mathrm{S}$ = salage,
$\mathrm{A}=$ affinage,
$\mathrm{AC}=$ affinage court,
$\mathrm{AL}=$ affinage long.

Fabrication avec maturation du lait à $12^{\circ} \mathrm{C}-15^{\circ} \mathrm{C}$ après la pasteurisation.

* Fabrication sans maturation.

développe et atteint au moment de l'emprésurage un nombre de bactéries par ml de lait égal ou supérieur à $1.10^{8}$ ce qui entraîne aux stades suivants de la fabrication une acidification rapide avec abaissement du $\mathrm{pH}$ du caillé jusqu'à 4,9-5,0 environ, $4 \mathrm{~h}$ après l'emprésurage. Une telle maturation s'étant révélée particulièrement favorable au développement des bactéries coliformes dans le lait, les huit essais suivants ont été réalisés sans maturation du lait, qui était pasteurisé et ensemencé juste avant l'emprésurage à l'aide d'une suspension concentrée de streptocoques lactiques $(0,01$ à 0,1 p. 100 suivant la vitesse d'acidification recherchée).

Deux types d'affinage et de conservation ont été comparés :

a) affinage « court » de 10 à $11 \mathrm{j}$ à $12^{\circ} \mathrm{C}$, suivi d'une conservation à $4^{\circ} \mathrm{C}$ pendant $70 \mathrm{j}$, 
b) affinage " long " de 20 à $22 \mathrm{j}$ à $12^{\circ} \mathrm{C}$, suivi d'une conservation à $4^{\circ} \mathrm{C}$ pendant $60 \mathrm{j}$.

Cette longue conservation des échantillons de fromages (jusqu'à $80 \mathrm{j}$ après l'emprésurage) a été adoptée pour refléter les conditions de conservation qui peuvent se rencontrer dans le cas des fromages destinés à l'exportation (conservation avant expédition, transport, commercialisation).

\section{2) PRÉLÈVEMENT ET PRÉPARATION DES ÉCHANTILLONS}

Lait : à chaque fabrication, des échantillons étaient prélevés sur le lait après la pasteurisation et juste avant l'emprésurage, en vue du dénombrement des bactéries coliformes.

Fromage : des prélèvements étaient effectués sur le caillé $3 \mathrm{~h}$ et $6 \mathrm{~h}$ après l'emprésurage, sur le fromage $24 \mathrm{~h}$ et $48 \mathrm{~h}$ après l'emprésurage, puis sur les fromages âgés de $6 \mathrm{j}, 10 \mathrm{j}$ et ensuite tous les $10 \mathrm{j}$.

Chaque échantillon de fromage consistait en un fromage entier qui était partagé en deux parties égales, sans être écroûté. Une moitié du fromage servait à la détermination du $\mathrm{pH}$. Pour cela le demi-fromage, placé dans un sac plastique, était malaxé soigneusement à la main et le $\mathrm{pH}$ de la pâte obtenue était déterminé à l'aide d'une électrode combinée pointue (pHmètre Metrohm). L'autre moitié du fromage, destinée au dénombrement des cơliformes, était pesée dans le bol (stérilisé à l'autoclave) d'un mixer « Waring

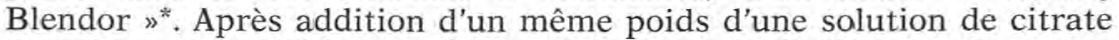
de sodium à 2 p. 100, le mélange était homogénéisé à grande vitesse pendant $1 \mathrm{mn}$, puis dilué au $1 / 5$ avec la même solution de citrate. La suspension ainsi obtenue (dilution de fromage au 1/10) servait à préparer les dilutions décimales qui étaient faites dans l'eau peptonée à 0,1 p. 100 .

\section{3) DÉNOMBREMENT DES BACTÉRIES COLIFORMES}

Lait : un dénombrement était effectué sur gélose au désoxycholate (Désoxycholate lactose agar Difco 0.420.01) avec incubation des boîtes de Petri à $37^{\circ} \mathrm{C}$ pendant $20 \mathrm{~h}$.

Pour les échantillons peu contaminés en coliformes la méthode suivante de détermination du nombre le plus probable (MPN) était utilisée :

\footnotetext{
* Cette méthode, consistant à utiliser un demi-fromage pour l'examen bactériologique a été dictée par la constatation, au cours d'essais précédents, d'une très grande hétérogénéité dans la répartition des coliformes dans la masse du fromage, hétérogénéité d'autant plus grande que le fromage était peu contaminé en coliformes.
} 
Trois flacons contenant $100 \mathrm{ml}$ de milieu bilié au vert brillant à double concentration (Brillant green bile 2 p. 100 Difco 0007.01) et trois tubes contenant $10 \mathrm{ml}$ du même milieu étaient additionnés des mêmes quantités de lait $(100 \mathrm{ml}$ et $10 \mathrm{ml}$ respectivement) de façon à réaliser un enrichissement du lait en bactéries coliformes. Après incubation pendant $24 \mathrm{~h}$ à $30^{\circ} \mathrm{C}$, une öse de chacun des flacons et des tubes était ensemencée dans des tubes de milieu au vert brillant à simple concentration contenant une cloche de Durham. Ces tubes étaient incubés pendant $48 \mathrm{~h}$ à $30^{\circ} \mathrm{C}$, et tout dégagement de gaz était considéré comme indice de la présence de bactéries coliformes dans les flacons et tubes de lait correspondants.

On ensemençait par ailleurs trois tubes de milieu au vert brillant à simple concentration avec chacun $1 \mathrm{ml}$ de lait suivant la technique habituelle.

Cette méthode permettait de déceler les bactéries coliformes en nombre égal ou supérieur à trois germes par litre de lait.

Fromage: un dénombrement était effectué comme pour le lait sur gélose au désoxycholate avec incubation des boîtes de Petri à $37^{\circ} \mathrm{C}$ pendant $24 \mathrm{~h}$.

Pour les échantillons peu contaminés (moins de dix germes par g) on utilisait là encore une méthode de détermination du nombre le plus probable :

Trois tubes contenant $10 \mathrm{ml}$ de milieu au vert brillant à double concentration étaient additionnés chacun de $10 \mathrm{ml}$ de la suspension de fromage. Après incubation à $37^{\circ} \mathrm{C}$ pendant $24 \mathrm{~h}$, une öse de chacun des tubes était ensemencée dans des tubes de milieu au vert brillant à simple concentration avec cloches de Durham.

D'autre part deux séries de trois tubes de milieu au vert brillant à simple concentration étaient ensemencées respectivement avec $1 \mathrm{ml}$ et $0,1 \mathrm{ml}$ de la suspension de fromage.

Cette méthode permettait, en utilisant la table de Mac Crady, de déceler les bactéries coliformes en nombre égal ou supérieur à trente germes pour $100 \mathrm{~g}$ de fromage.

\section{4) ISOLEMENT ET IDENTIFICATION DES BACTERIES COLIFORMES}

A différentes reprises les colonies rouges développées sur le milieu gélosé au désoxycholate ont été repiquées et les souches isolées ont été identifiées par la micro-méthode "API » destinée à l'identification des Enterobacteriaceae*. L'exactitude de cette méthode a été évaluée par Washington II et al. [3].

\footnotetext{
* API System, La Balme-les-Grottes - 38390 Montalieu-Vercieu.
} 


\section{Résultats}

La figure 2 montre à titre d'exemple les résultats obtenus au cours de quatre essais effectués avec maturation du lait à $15^{\circ} \mathrm{C}$

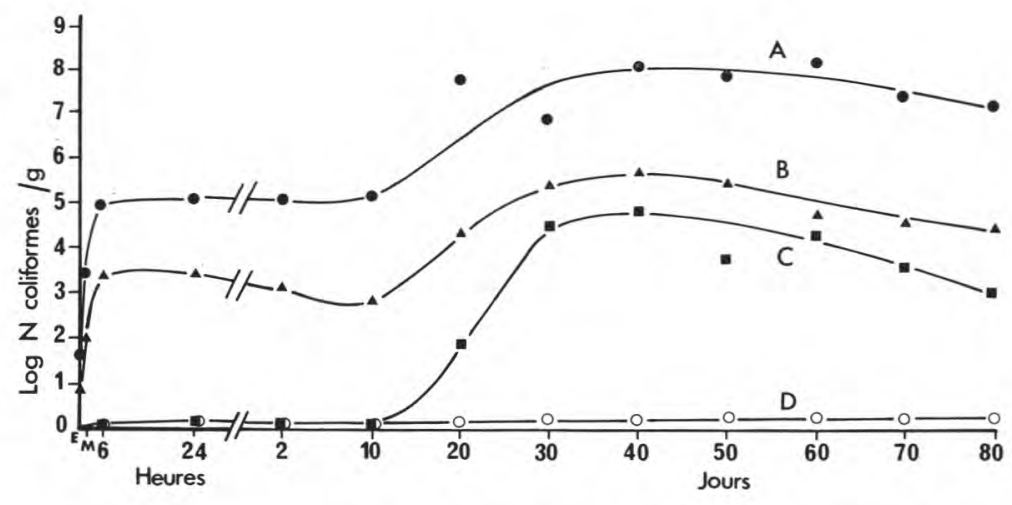

fig. 2

Développement des bactéries coliformes dans le fromage de Camembert au cours de la fabrication, de l'affinage $\left(10 \mathrm{j}\right.$ à $\left.12^{\circ} \mathrm{C}\right)$ et de la conservation au froid $\left(4^{\circ} \mathrm{C}\right)$.

$\mathrm{E}=$ emprésurage, $\mathrm{M}=$ mise en moules.

(essais A, B, C) ou sans maturation (essai D), l'affinage étant réalisé selon les conditions décrites en (a). Dans l'essai A le lait contenait au moment de l'emprésurage soixante coliformes par ml, dans l'essai B huit coliformes par $\mathrm{ml}$, dans l'essai $\mathrm{C}$ il contenait moins d'un coliforme par $\mathrm{ml}$ (probablement de l'ordre d'un coliforme pour $10 \mathrm{ml}$ ) et dans l'essai D moins de trois coliformes par litre de lait.

On observe sur les courbes A et B un développement rapide des coliformes dès l'emprésurage et au cours des 6 premières heures de la fabrication, suivi d'un palier ou même d'une diminution de leur nombre après $24 \mathrm{~h}$. Entre le $10^{\mathrm{e}}$ et le $30^{\mathrm{e}} \mathrm{j}$, une nouvelle croissance apparaît, la population maximale atteignant $10^{5}$ (courbe B) et $10^{8}$ (courbe A) coliformes par g de fromage. A ce stade la flore coliforme dominante est constituée par Enterobacter hafniae* (Hafnia alvei [4]).

\footnotetext{
* Nous remercions très vivement le docteur Le Minor et ses collaborateurs de l'Institut Pasteur de Paris, qui ont bien voulu confirmer l'identification des souches $d^{\prime}$ 'Ent. hafniae.
} 
Dans l'essai C, on n'observe aucun développement décelable de coliformes pendant la fabrication proprement dite (24 premières heures) ni pendant l'affinage à $12^{\circ} \mathrm{C}$, mais un développement important se manifeste pendant la conservation des fromages à $4^{\circ} \mathrm{C}$. La flore dominante est également constituée par Ent. hafniae.

Enfin dans l'essai $\mathrm{D}$ la fabrication a été réalisée à partir d'un lait qui contenait moins de trois coliformes par litre de lait. Ce résultat était obtenu en supprimant la période de maturation du lait entre la pasteurisation du lait et l'emprésurage. Dans ce cas on n'observe aucun développement de coliformes, ni pendant la fabrication, ni pendant l'affinage et la conservation au froid des fromages.

Des déterminations du $\mathrm{pH}$ des fromages en cours d'affinage ont permis de constater que, lorsqu'on observait un développement de coliformes en cours d'affinage, ce développement coïncidait avec une élévation du $\mathrm{pH}$ qui passait progressivement de 4,6-4,7 (fromage de 10 j) à 6,7-7,0 (fromages âgés de 40 j) (fig. 3).

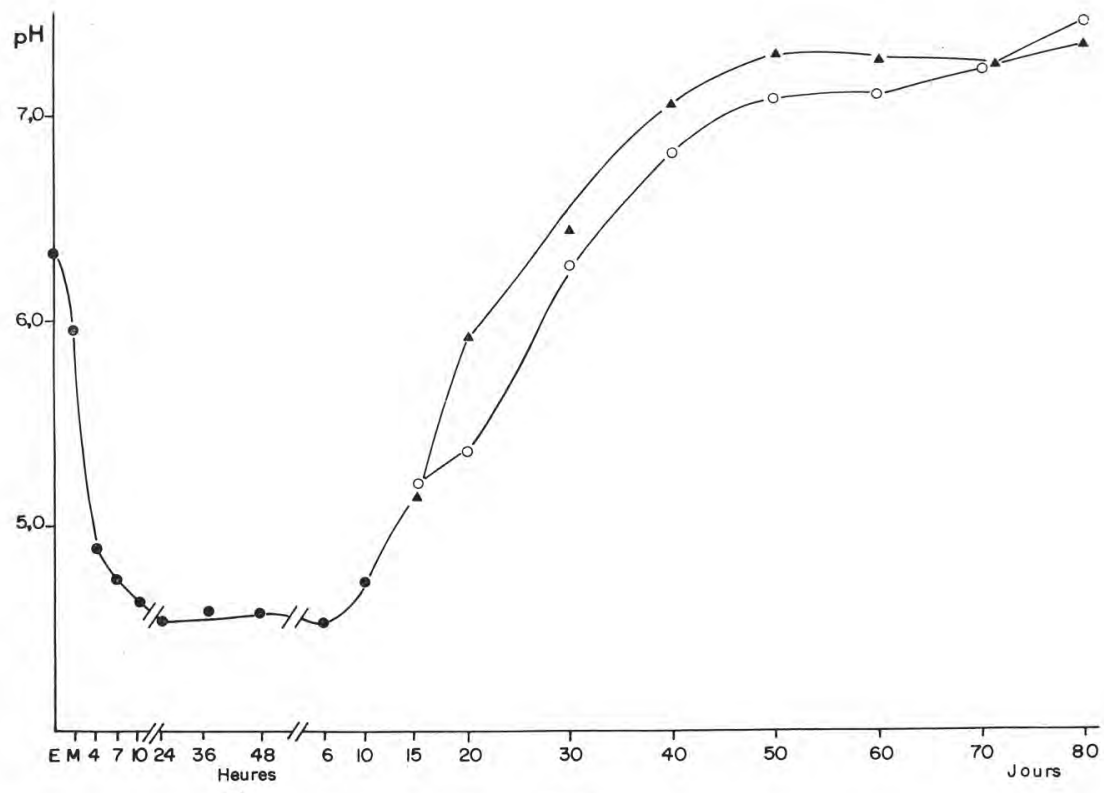

fig. 3

Evolution du $\mathrm{pH}$ dans le lait, le caillé et le fromage au cours de la fabrication et l'affinage des fromages expérimentaux.

$$
\mathrm{E}=\text { emprésurage, } \mathrm{M}=\text { mise en moules. }
$$

$\mathrm{O} \longrightarrow$ Fromages affinés pendant $10 \mathrm{j}$ à $12^{\circ} \mathrm{C}$ puis conservés à $4^{\circ} \mathrm{C}$.

ــ Fromages affinés pendant $20 \mathrm{j}$ à $12^{\circ} \mathrm{C}$ puis conservés à $4^{\circ} \mathrm{C}$. 
Dans le cas où l'on avait soumis les fromages à un affinage "long " $\left(20\right.$ à $22 \mathrm{j}$ à $\left.12^{\circ} \mathrm{C}\right)$ avant la conservation à $4^{\circ} \mathrm{C}$, on constatait à partir du $10^{\mathrm{e}}$ jour de fabrication, un développement rapide des coliformes, développement nettement plus rapide que celui qui avait été observé pour les fromages à affinage "court ". Le nombre maximum de coliformes atteint en cours d'affinage et de conservation représentait dans le cas de l'affinage "long " environ quinze fois le nombre maximum atteint dans le cas de l'affinage « court ».

II. - UNE SECONDE SERIE D'ESSAIS, comportant vingt-deux fabrications a été réalisée, également à la Laiterie Expérimentale de Jouy-en-Josas, en utilisant du lait exempt de bactéries coliformes (lait pasteurisé non maturé), qui était alors ensemencé avec différentes souches de coliformes:

- une souche d'Ent. hafniae : quatre fabrications,

- une souche d'E. coli isolée d'un fromage affiné : six fabrications,

- une souche d'E. coli entéropathogène du sérotype 0124:B17 (souche fermentant le lactose)* : cinq fabrications,

- une souche d'E. coli entéropathogène du sérotype 0128:B12 (souche fermentant également le lactose)* : deux fabrications,

- un mélange, en différentes proportions d'Ent. hafniae et d'E. coli 0128:B12 : cinq fabrications.

\section{Méthodes}

Les techniques de fabrication et les conditions d'affinage étaient les mêmes que dans la première série d'essais. Chaque fabrication d'essai (lait ensemencé avec une souche déterminée de coliformes) était couplée avec une fabrication témoin (c'est-à-dire ne comportant pas d'ensemencement avec la souche de coliformes) de façon à être en mesure de vérifier si un développement " accidentel " de bactéries coliformes s'était ou non produit pendant la fabrication et l'affinage.

Le dénombrement des bactéries coliformes (Ent. hafniae et $E$. coli) était effectué sur milieu gélosé au désoxycholate de sodium, les boîtes de Petri étaient incubées à $37^{\circ} \mathrm{C}$. Dans le cas des fabrications où le lait était ensemencé avec les deux germes simultanément (Ent. hafniae et E. coli), deux séries de boîtes de Petri étaient ensemencées, une série était incubée à $44^{\circ} \mathrm{C}$ pendant $22 \mathrm{~h}$ et servait au dénombrement d'E. coli, l'autre série était incubée pendant $10 \mathrm{j}$ à $7^{\circ} \mathrm{C}-8^{\circ} \mathrm{C}$ pour le dénombrement d'Ent. hafniae. On a vérifié avec

\footnotetext{
* Nous remercions le docteur J.C. Olson, Division de Microbiologie, F.D.A., Washington D.C. (U.S.A.), pour la fourniture des souches d'E. coli entéropathogènes.
} 
chacune des souches prises séparément qu'E. coli ne formait pas de colonies à $7^{\circ} \mathrm{C}-8^{\circ} \mathrm{C}$ pendant 10 jours et qu'Ent. hafniae ne formait pas de colonies au bout de $22 \mathrm{~h}$ à $44^{\circ} \mathrm{C}$.

\section{Résultats}

Les figures 4, 5 et 6 montrent l'évolution des populations d'Ent. hafniae (fig. 4) et d'E. coli (fig. 5 et 6) dans les fromages de trois

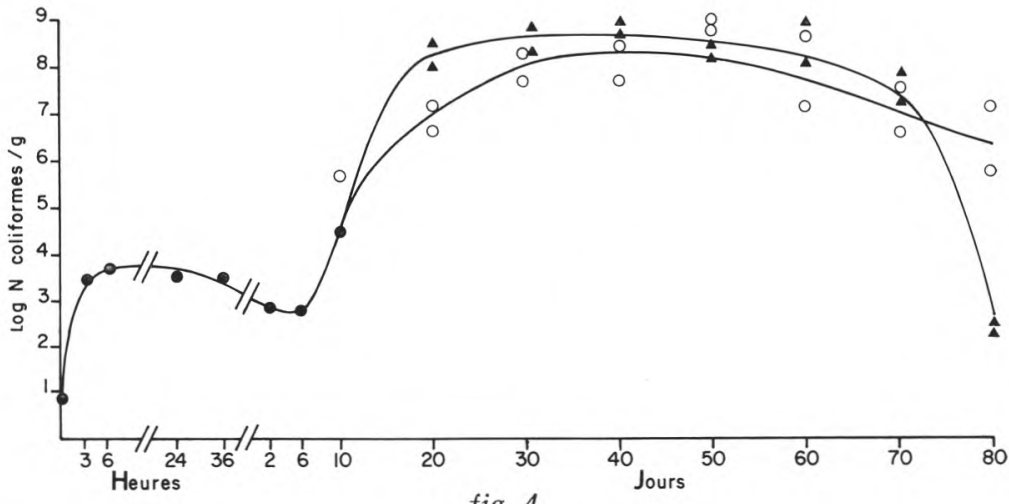

fig. 4

Développement de Ent. hafniae dans le fromage de Camembert.

$\bigcirc-0$ Fromages affinés pendant $10 \mathrm{j}$ à $12^{\circ} \mathrm{C}$ puis conservés à $4^{\circ} \mathrm{C}$.

$\Delta$ _ـ Fromages affinés pendant $20 \mathrm{j}$ à $12^{\circ} \mathrm{C}$ puis conservés à $4^{\circ} \mathrm{C}$.

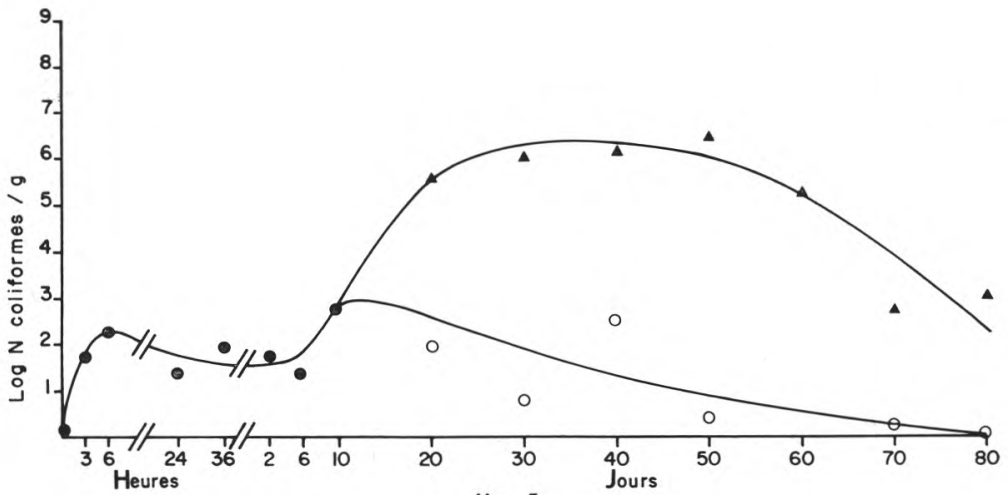

fig. 5

Développement de E. coli dans le fromage de Camembert.

○— O- Fromages affinés pendant $10 \mathrm{j}$ à $12^{\circ} \mathrm{C}$ puis conservés à $4^{\circ} \mathrm{C}$.

$\Delta-\mathbf{\Delta}$ Fromages affinés pendant $20 \mathrm{j}$ à $12^{\circ} \mathrm{C}$ puis conservés à $4^{\circ} \mathrm{C}$. 


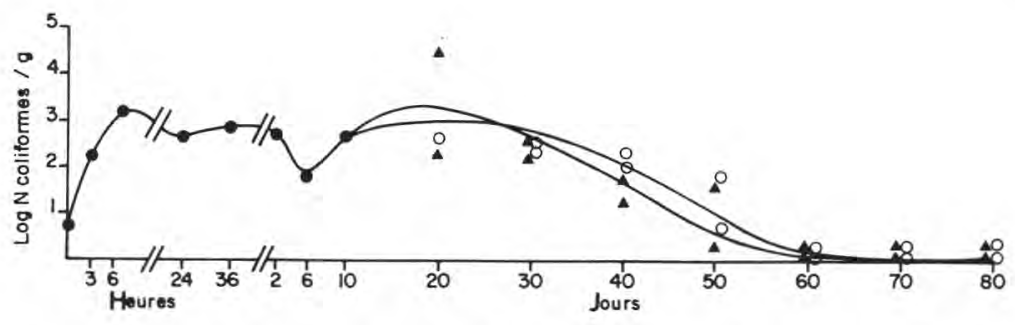

fig. 6

Développement d'une souche de $E$. coli entéropathogène dans le fromage de Camembert.

0 Fromages affinés pendant $10 \mathrm{j}$ à $12^{\circ} \mathrm{C}$ puis conservés à $4^{\circ} \mathrm{C}$.

$\Delta-\Delta$ Fromages affinés pendant $20 \mathrm{j}$ à $12^{\circ} \mathrm{C}$ puis conservés à $4^{\circ} \mathrm{C}$.

fabrications choisies en exemple et dans lesquelles la vitesse d'acidification était plus lente que dans les essais précédents (inoculation du lait avec 0,01 p. 100 d'une suspension concentrée de streptocoques lactiques sans maturation prolongée et avec abaissement du $\mathrm{pH}$ à $5,8-5,9,4 \mathrm{~h}$ après l'emprésurage). Pour les trois fabrications le nombre de germes inoculés dans le lait était faible ( 8 germes $/ \mathrm{ml}$ dans le cas d'Ent. hafniae, 1,4 et 7 dans le cas d'E. coli).

On voit que, même en partant d'un nombre de germes très faible dans le lait, les populations de coliformes atteintes dans les fromages au cours de l'affinage et de la conservation au froid peuvent atteindre des niveaux très élevés : $10^{8}$ à $10^{\circ}$ dans le cas d'Ent. hafniae, $10^{6}$ dans le cas d'E. coli (souche isolée de fromage). Les souches d'E. coli entéropathogènes, 0124:B17 et 0128:B12, se sont relativement peu développées (fig. 6) au cours de l'affinage (elles ne dépassent guère $10^{3}$ par g). De plus ces germes ont eu tendance à diminuer plus ou moins rapidement au cours de la conservation des fromages au froid et en général on ne les a plus retrouvés dans les fromages âgés de plus de $60 \mathrm{j}$.

Pour étudier l'influence d'un abaissement plus ou moins rapide du $\mathrm{pH}$ au cours de la fabrication sur le développement des bactéries coliformes dans les fromages, plusieurs essais ont été réalisés en utilisant une dose importante de levain $(0,05$ ou 0,1 p. 100 d'une suspension concentrée de streptocoques lactiques). L'abaissement de $\mathrm{pH}$ entre l'inoculation simultanée des coliformes et du levain et le prélèvement effectué $3 \mathrm{~h}$ après l'emprésurage était alors de 1,6 à 1,8 unité $\mathrm{pH}(\mathrm{pH}$ voisin de $4,8-5,0)$.

Les figures 7 et 8 montrent que, dans ce cas, lorsque le lait est inoculé avec des nombres importants de coliformes $\left(4.10^{4}\right.$ germes $\left./ \mathrm{ml}\right)$, l'acidification rapide du caillé entraîne une diminution relativement 


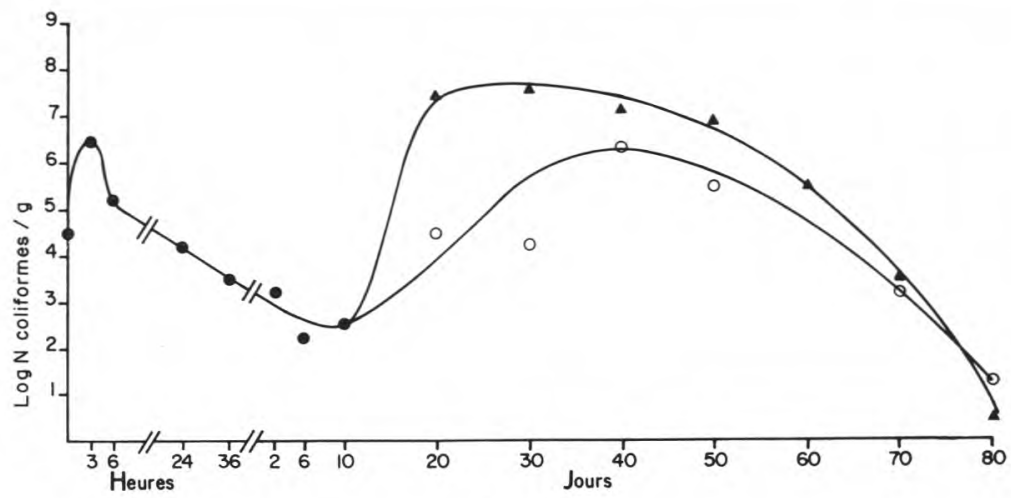

fig. 7

Développement de Ent. hafniae, dans le fromage de Camembert. Lait ensemencé avec 40000 germes $/ \mathrm{ml}$ et acidification rapide.

○—— - Fromages affinés pendant $10 \mathrm{j}$ à $12^{\circ} \mathrm{C}$ puis conservés à $4^{\circ} \mathrm{C}$.

$\mathbf{\Delta}$ Fromages affinés pendant $20 \mathrm{j}$ à $12^{\circ} \mathrm{C}$ puis conservés à $4^{\circ} \mathrm{C}$

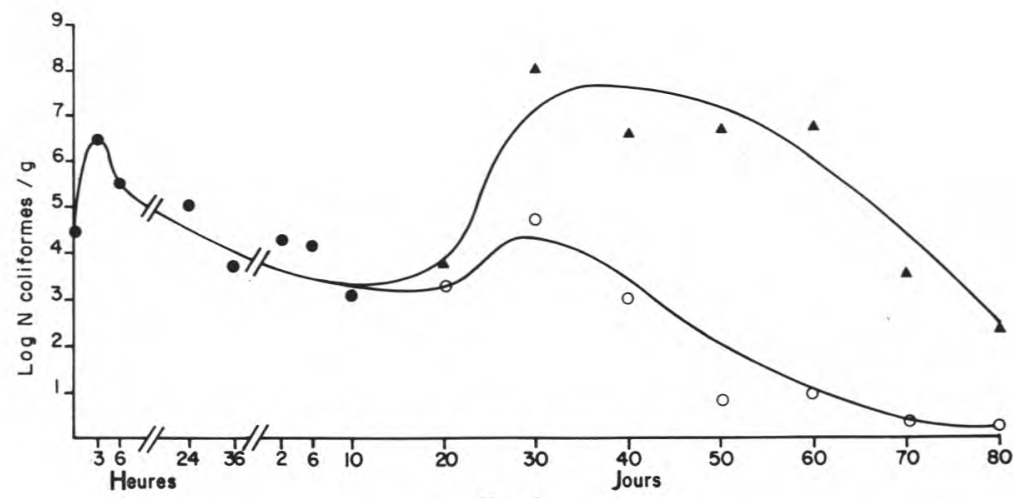

fig. 8

Développement de E. coli dans le fromage de Camembert. Lait ensemencé avec 40000 germes $/ \mathrm{ml}$ et acidification rapide.

○—— Fromages affinés pendant $10 \mathrm{j}$ à $12^{\circ} \mathrm{C}$ puis conservés à $4^{\circ} \mathrm{C}$.

$\Delta$ - $\mathbf{\Delta}$ Fromages affinés pendant $20 \mathrm{j}$ à $12^{\circ} \mathrm{C}$ puis conservés à $4^{\circ} \mathrm{C}$.

importante du nombre des coliformes pendant la fabrication et jusqu'au $10^{\mathrm{e}}$ jour, mais on observe cependant ultérieurement une augmentation marquée du nombre de ces germes au cours de l'affinage. Une acidification rapide du caillé ne permet donc pas de maîtriser le développement des coliformes dans le fromage lorsque ces germes sont en nombre élevé dans le lait de fabrication, comme. on l'avait déjà constaté dans la première série d'essais. 
Les essais réalisés à partir de lait inoculé simultanément avec E. coli entéropathogène 0128:B12 et Ent. hafniae (fig. 9) ont montré

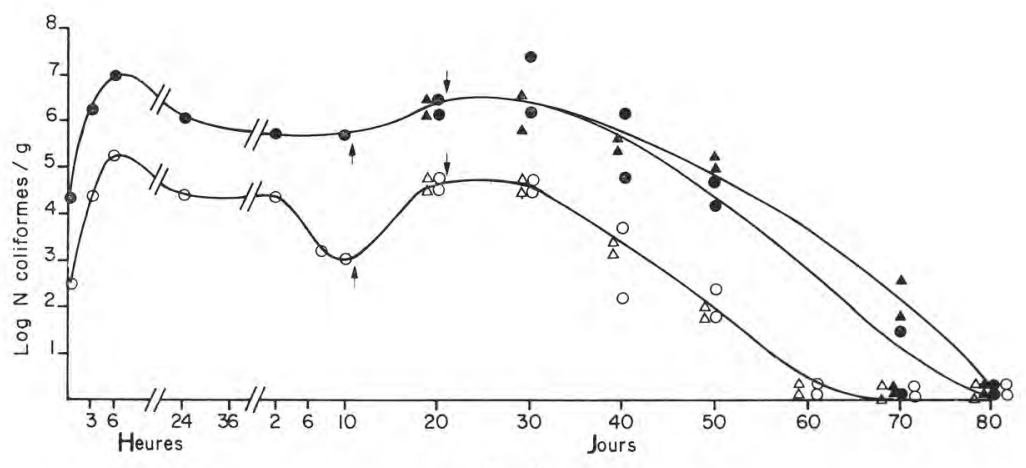

fig. 9

Développement simultané de Ent. hafniae et de E. coli 0.128:B12 dans le fromage de Camembert.

$\mathrm{O}-\mathrm{O}$ Fromages affinés pendant $10 \mathrm{j}$ à $12^{\circ} \mathrm{C}$ puis conservés à $4^{\circ} \mathrm{C}$.

$\triangle \longrightarrow \triangle$ Fromages affinés pendant $20 \mathrm{j}$ à $12^{\circ} \mathrm{C}$ puis conservés à $4^{\circ} \mathrm{C}$.

- - Fromages affinés pendant $10 \mathrm{j}$ à $12^{\circ} \mathrm{C}$ puis conservés à $4^{\circ} \mathrm{C}$.

$\mathbf{A}$ Fromages affinés pendant $20 \mathrm{j}$ à $12^{\circ} \mathrm{C}$ puis conservés à $4^{\circ} \mathrm{C}$.

E. coli

Ent, hafniae

qu'E. coli 0128:B12 suit une évolution comparable à celle observée dans les essais où il est seul (fig. 6) tandis qu'Ent. hafniae semble être relativement inhibé : en effet, le nombre d'Ent. hafniae augmente assez peu au cours de l'affinage et de la conservation au froid, si on le compare aux nombres atteints dans les essais où cet organisme est seul (fig. 4 et 7 ).

Dans cette série d'essais, il est important de noter que, parallèlement aux vingt-deux fabrications qui comportaient une inoculation de germes coliformes, correspondaient vingt-deux fabrications témoins pour lesquelles les mêmes prélèvements ont été effectués depuis le lait emprésuré jusqu'à la fin de la conservation. Ces vingtdeux fabrications ont été toutes trouvées indemnes de coliformes et d'E. coli.

III. - LA TROISIEME SERIE D'ESSAIS était constituée par onze fabrications industrielles de Camembert. 


\section{Méthodes}

Les fabrications étaient menées dans les conditions habituelles de l'usine. Cependant un soin particulier était pris au niveau du nettoyage et de la désinfection du matériel de façon à se placer dans des conditions de propreté aussi bonnes que possible.

Le lait était chauffé à $70^{\circ} \mathrm{C}$ pendant $30 \mathrm{~s}$, puis " maturé " à $8^{\circ} \mathrm{C}$ pendant $16 \mathrm{~h}$ avec $0,5 \mathrm{p} .100$ de ferments mésophiles (Flora Danica). Le lait était réchauffé ensuite à $32,5^{\circ} \mathrm{C}$, ensemencé avec 2 p. 100 de ferments Flora Danica et emprésuré immédiatement.

Les fromages étaient affinés pendant 9 ou $10 \mathrm{j}$ à $12^{\circ} \mathrm{C}$ puis expédiés au Laboratoire de Jouy-en-Josas. Dès réception les fromages étaient répartis en deux lots, dont l'un était conservé à $4^{\circ} \mathrm{C}$, l'autre à $8^{\circ} \mathrm{C}$.

Des échantillons de lait, de caillé, et de fromage ont été prélevés comme précédemment aux différents stades de la fabrication et de l'affinage.

Les dénombrements de coliformes étaient effectués par ensemencement sur gélose au désoxycholate avec incubation des boîtes de Petri à $37^{\circ} \mathrm{C}$ pendant $24 \mathrm{~h}$.

On dénombrait d'autre part les " coliformes fécaux » par la méthode de Mc Kenzie et al. décrite dans Thatcher et Clark [5].

Les échantillons étaient ensemencés sur bouillon de Mc Conkey à $37^{\circ} \mathrm{C}$ (trois tubes par dilution). Les tubes présentant une production de gaz après $24 \mathrm{~h}$ et $48 \mathrm{~h}$ étaient réensemencés d'une part sur bouillon bilié lactosé au vert brillant et d'autre part sur eau peptonée. Des réactions positives (production de gaz dans le milieu au vert brillant et production d'indole dans l'eau peptonée) étaient considérées comme indices de la présence de «coliformes fécaux».

\section{Résultats}

La figure 10 rassemble les résultats obtenus dans le cas des fromages conservés à $4^{\circ} \mathrm{C}$ après leur affinage de $10 \mathrm{j}$ à $12^{\circ} \mathrm{C}$, et la figure 11, les résultats dans le cas des fromages conservés à $8^{\circ} \mathrm{C}$, après leur affinage de $10 \mathrm{j}$ à $12^{\circ} \mathrm{C}$.

Le lait de fabrication contenait en général moins de 100 bactéries coliformes par $\mathrm{ml}$ avec une proportion très variable de coliformes fécaux.

A la fin de la fabrication (démoulage) et pendant l'affinage à $12^{\circ} \mathrm{C}$ (jusqu'au $10^{\mathrm{e}} \mathrm{j}$ ), le nombre des coliformes totaux était compris entre 100 et 200 par g, celui des coliformes fécaux était légèrement inférieur.

C'est à partir du $10^{\mathrm{e}} \mathrm{j}$ que l'on voit le nombre de coliformes totaux augmenter de façon importante, atteignant $10^{5}$ à $10^{6}$ (il s'agit là encore essentiellement d'une flore d'Ent. hafniae), tandis que le 


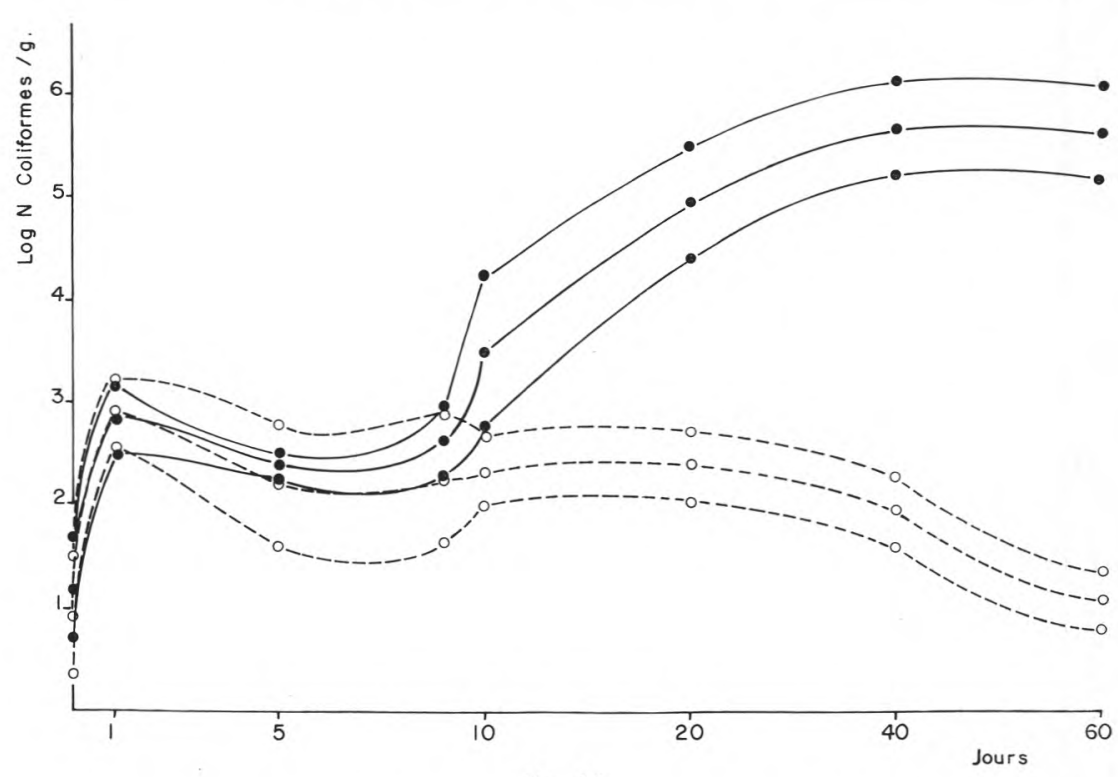

fig. 10

Développement des bactéries coliformes ( - — ) et des coliformes fécaux $(\mathrm{O}-\mathrm{O})$ dans onze fabrications industrielles de fromages de type Camembert, affinés pendant $10 \mathrm{j}$ à $12^{\circ} \mathrm{C}$ puis conservés à $4^{\circ} \mathrm{C}$. Courbe moyenne et intervalle de confiance.

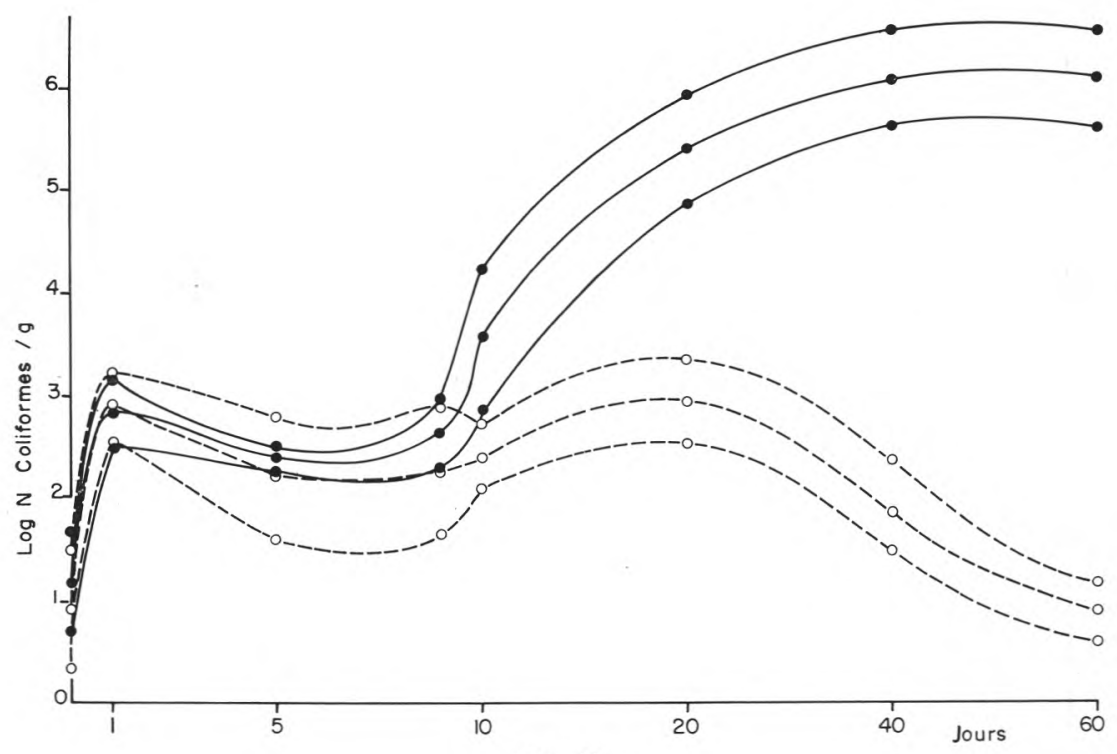

fig. 11

Développement des bactéries coliformes fécaux $(\mathrm{O}-\mathrm{O})$ dans onze fabrications industrielles de fromages de type Camembert, affinés pendant $10 \mathrm{j}$ à $12^{\circ} \mathrm{C}$ puis conservés à $8^{\circ} \mathrm{C}$. Courbe moyenne et intervalle de confiance. 
nombre de coliformes fécaux diminue progressivement. Cependant à $60 \mathrm{j}$, les fromages contiennent encore en moyenne une dizaine de coliformes fécaux par $\mathrm{g}$.

\section{DISCUSSION ET CONCLUSIONS}

La première série d'essais réalisée à la Laiterie Expérimentale de Jouy-en-Josas (fromages contaminés naturellement par des coliformes), de même que les fabrications industrielles ont montré qu'une contamination même faible du lait en bactéries coliformes peut entraîner au cours de l'affinage des fromages à pâte molle une multiplication de ces coliformes jusqu'à un niveau difficilement compatible avec les normes d'hygiène.

Un premier développement important des coliformes se produit au début de la fabrication, avant que le $\mathrm{pH}$ n'ait suffisamment diminué du fait du développement du levain lactique. Une acidification rapide et forte détruit une partie importante de cette flore coliforme mais elle ne suffit pas à assainir complètement le fromage. Par la suite, lorsqu'au cours de l'affinage le $\mathrm{pH}$ remonte, on assiste à une nouvelle multiplication des coliformes qui peuvent atteindre des nombres élevés ( $10^{6}$ à $10^{8}$ dans certains cas).

Ce développement des bactéries coliformes dans le fromage (Gouda, Cheddar) a déjà fait l'objet d'études qui ont bien mis en évidence le rôle du $\mathrm{pH}$ dans ce développement $[6,7,8,9]$.

D'une façon générale nous avons observé une bonne relation entre le nombre de coliformes présents dans le lait et le nombre maximum de coliformes trouvés soit au cours de la fabrication, soit au cours de l'affinage. En effet, en rangeant les différents essais d'une part suivant le nombre de bactéries coliformes à l'emprésurage, d'autre part suivant le nombre maximum de bactéries coliformes trouvées dans le fromage au cours de l'affinage, nous avons effectué le test de Spearman [10] pour mesurer la corrélation entre ces deux variables.

\begin{tabular}{|c|c|c|c|}
\hline & \multicolumn{3}{|c|}{ Conditions d'affinage } \\
\hline & $\begin{aligned} & 10 \mathrm{j} \text { à } 12^{\circ} \mathrm{C} \\
+ & \text { séjour à } 4^{\circ} \mathrm{C}\end{aligned}$ & $\begin{aligned} & 10 \mathrm{j} \text { à } 12^{\circ} \mathrm{C} \\
+ & \text { séjour à } 8^{\circ} \mathrm{C}\end{aligned}$ & $\begin{aligned} & 20 \mathrm{j} \text { à } 12^{\circ} \mathrm{C} \\
+ & \text { séjour à } 4^{\circ} \mathrm{C}\end{aligned}$ \\
\hline Nombre d'essais & 36 & 25 & 25 \\
\hline$r_{s}$ (coefficient de Spearman) & $\because \quad 0,710$ & 0,781 & 0,812 \\
\hline $\mathrm{t}$ & & $5,99 * *$ & $6,67^{* *}$ \\
\hline
\end{tabular}


On constate que dans les trois conditions d'affinage, les valeurs de $t$ sont hautement significatives, ce qui montre que le nombre maximum de coliformes trouvés dans le fromage en cours d'affinage dépend très fortement du nombre de ces germes dans le lait de fabrication. Des observations semblables ont été faites pour le fromage de Cheddar par Dommett [8].

Il n'est pas impossible de fabriquer des fromages à pâte molle sans coliformes ainsi que l'ont montré les essais réalisés à la Laiterie Expérimentale de Jouy. Cependant les précautions habituelles d'hygiène ne suffisent pas à obtenir ce résultat. C'est en supprimant la période de maturation du lait que nous avons pu obtenir des fromages sans coliformes (à partir de lait contenant moins de trois germes par litre). Cependant dans la pratique industrielle le même résultat ne peut être escompté qu'avec des installations spécialement conçues en vue de faciliter le nettoyage et la désinfection.

Les coliformes qui se développent au cours de l'affinage et de la conservation au froid des fromages à pâte molle appartiennent en majorité à l'espèce Ent. hafniae. Ces bactéries sont connues pour leur aptitude à se développer à température relativement basse et doivent être considérées comme des bactéries psychrotrophes. Il est intéressant de noter que les souches que nous avons isolées étaient capables de fermenter le lactose, caractère considéré comme relativement rare dans cette espèce $[4,11,12]$.

Les essais de fabrication réalisés à la Laiterie Expérimentale de Jouy-en-Josas en ensemençant le lait avec des souches déterminées de coliformes ont montré qu'E. coli se développe moins vite et moins abondamment, et disparaît plus vite, qu'Ent. hafniae lorsque les fromages sont maintenus à $4^{\circ} \mathrm{C}$. Les souches d'E. coli entéropathogènes en particulier montraient une diminution constante au cours de la conservation des fromages à $4^{\circ} \mathrm{C}$. Nos résultats concordent bien avec les observations de Park et al. [9] qui, dans des conditions expérimentales comparables aux nôtres, notaient un maximum dans le développement des $E$. coli entéropathogènes $6 \mathrm{~h}$ après l'emprésurage, puis une diminution de leur nombre au cours de l'affinage, diminution d'autant plus rapide que le levain utilisé était plus acidifiant.

Compte tenu de ces résultats, il est vraisemblable que lorsque des fromages à pâte molle renferment à la fin de leur période d'affinage des $E$. coli (entéropathogènes ou non) ces fromages ont dû être le siège dans les premières heures de leur fabrication d'un développement considérable de ces germes qui devaient déjà être présents en grand nombre dans le lait de fabrication.

Les méthodes habituelles de dénombrement des coliformes ne permettent pas de détecter les souches d'E. coli ne fermentant pas le lactose. Or on sait que certaines souches d'E. coli entéropathogènes, appartenant en particulier au sérotype 0124:B17, ne fer- 
mentent pas ce sucre. Des méthodes permettant de rechercher ces germes dans le fromage ont été décrites récemment $[13,14,15]$.

Enfin il faut rappeler que nos résultats ont été obtenus dans le cas d'une technologie de type traditionnel. Le cas de fabrications mécanisées qui impliquent souvent une acidification moindre et parfois un "recyclage " du lactosérum à une température très favorable à la croissance des bactéries et qui font appel d'autre part à un matériel a priori plus difficile à nettoyer et à désinfecter que le traditionnel, devrait faire l'objet de recherches complémentaires.

Compte tenu des observations qui précèdent, quels conseils peuton donner aux fromagers qui fabriquent le Camembert ?

$1^{\circ}$ Réduire à un niveau aussi bas que possible les contaminations du lait de fromagerie après pasteurisation. Si l'on peut atteindre moins de trois coliformes par litre de lait les chances de voir se produire un développement des coliformes seront faibles ou nulles. Même si un niveau aussi faible ne peut être atteint régulièrement à l'échelle industrielle il y a intérêt à s'en rapprocher le plus possible (par exemple en supprimant toute maturation du lait avant fabrication).

$2^{\circ}$ Effectuer le contrôle de la teneur en coliformes sur le caillé 4 à $6 \mathrm{~h}$ après l'addition de présure au lait, par exemple en inoculant trois fois $1 \mathrm{~g}$ de caillé dans trois tubes de milieu sélectif approprié liquide [16]. C'est à ce stade qu'une contamination même faible en coliformes sera la plus facile à révéler avec un inoculum relativement peu important, ne nécessitant par conséquent que des volumes de milieu de culture relativement faibles. En d'autres termes un contrôle effectué à ce stade précoce donnera à peu de frais l'indication la plus utile sur le développement ultérieur le plus vraisemblable des coliformes.

$3^{\circ}$ a) Raccourcir l'affinage à la durée minimum compatible avec une qualité convenable des fromages. A cet égard une durée de $10 \mathrm{j}$ (entre emprésurage et mise en frigorifique) est la période la plus courte qui ait été expérimentée dans ce travail. Peut-être est-il possible de la réduire encore un peu sans inconvénient appréciable ?

b) Aussitôt après l'affinage, placer les fromages à $4^{\circ} \mathrm{C}$ et les maintenir sans interruption à cette température. C'est de cette manière qu'on évitera le mieux le développement d'E. coli et notamment des $E$. coli entéropathogènes qui ne se développent d'une manière importante qu'à des températures plus élevées.

Pour des fromages maintenus à $4^{\circ} \mathrm{C}$, dans une chaîne de froid ininterrompue, le développement éventuel d'un certain nombre d'Ent. hafniae (coliformes psychrotrophes) ne comportera pas les mêmes risques que si le fromage est maintenu plus longtemps à des température voisine de - ou supérieures à $-10^{\circ} \mathrm{C}$. A $4^{\circ} \mathrm{C}$, le développement des $E$. coli entéropathogènes, à supposer qu'il existe, sera faible ou très faible et un nombre même assez grand d'Ent. hafniae n'aura, 
de ce fait, pas la même signification sur le plan de l'hygiène que si le fromage avait été maintenu à une température plus haute où le développement des $E$. coli entéropathogènes aurait pu devenir plus important.

\section{R és u m é}

1. On a suivi, au cours de seize fabrications expérimentale de fromages de type Camembert, les variations du nombre de bactéries coliformes présentes dans le lait de fabrication, dans le caillé et dans les fromages au cours de leur affinage et de leur conservation au froid. Une contamination même faible du lait en bactéries coliformes peut entraîner, au cours de l'affinage des fromages, une multiplication de ces coliformes jusqu'à un niveau difficilement compatible avec les normes d'hygiène. Les coliformes qui se développent au cours de l'affinage et de la conservation au froid appartiennent en majorité à l'espèce Enterobacter hafniae.

2. Vingt-deux fabrications expérimentales réalisées en ensemençant le lait de fabrication avec différentes souches d'Ent. hafniae et d'Escherichia coli (dont deux souches entéropathogènes) ont montré qu'E. coli se développe moins vite et moins abondamment, et disparaît plus vite, qu'Ent. hafniae lorsque les fromages sont maintenus à $+4^{\circ} \mathrm{C}$ après $10 \mathrm{j}$ d'affinage à $12^{\circ} \mathrm{C}$.

3. Dans onze fabrications industrielles de Camembert, on a constaté, comme en fabrication expérimentale, un développement important de bactéries coliformes au cours de l'affinage du fromage, le nombre de ces bactéries pouvant atteindre $10^{5}$ à $10^{6}$ par $\mathrm{g}$, lorsque le lait de fabrication contient moins de 100 bactéries coliformes par ml.

\section{S u m m a ry}

\section{ORIGIN AND DEVELOPMENT OF COLIFORM BACTERIA IN SOFT CHEESE}

1. 16 experimental batches of Camembert cheese were made in order to study the development of the coliform bacteria in the vat milk, in the curd and in the cheese during ripening and cold storage. It was seen that a contamination of the milk, however slight, by coliform bacteria may lead during ripening to a level which is far above a satisfactory standard of hygiène. Most of the coliforms which developed during ripening and cold storage were Enterobacter hafniae.

2. 22 experimental batches of cheese were made by inoculating the vat milk with different strains of Ent. hafniae and Escherichia coli (including 2 enteropathogenic strains). It was found that $E$. coli developed more slowly and disappeared more quickly than Ent. hafniae when the cheeses were stored at $4^{\circ} \mathrm{C}$ after 10 days ripening at $12^{\circ} \mathrm{C}$. 
3. In 11 batches of Camembert cheese made industrially it was seen, as in the experimental batches, than there was a considerable development of coliform bacteria during the ripening of the cheese reaching a level of $10^{5}$ to $10^{6}$ per gramme even when the vat milk contained no more than 100 coliforms per $\mathrm{ml}$.

Reçu pour publication en octobre 1976.

\section{Bibliographie}

[1] Schnurrenberger (L. W.), Beck (W. R.) and Pate (J.) (1971). - Gastro-enteritis attributed to imported French cheese. Morbidity mortality Weekly Report, 20, 427-428.

[2] Marier (R.), Wells (J. G.), Swanson (R.), Callahan (W.) and Mahlman (I. J.) (1973). - An outbreak of enteropathogenic E. coli foodborn disease traced to imported French cheese. Lancet, 1376-1378.

[3] Washington II (J.A.), Yu (Pauline K. W.) and Martin (W. J.) (1971). - Evaluation of accuracy of multitest micromethod system for identification of Enterobacteriaceae. Applied Microbiol., 22, 267-269.

[4] Bergey's manual of Determinative bacteriology, 8th Edition, The Williams \& Wilkins Co., Baltimore, 1974.

[5] Thatcher (F.S.) and CLARK (D. S.) (1968). - Microorganisms in foods : their significance and methods of enumeration. University of Toronto Press, p. 78.

[6] Fryer (T. F.) (1974). - Le contrôle des bactéries coliformes dans le Gouda. $X I X^{\circ}$ Congrès Intern. Laiterie, $1 \mathrm{~F}, 602-603$.

[7] Dommetт (T. W.) (1970). - Studies on coliform organisms in Cheddar cheese. Aust. J. Dairy Technol., 25, 54-60.

[8] Dommetт (T. W.) (1974). - Variations in levels of coliforms and Escherichia coli type 1 in Queensland Cheddar cheese. Aust. J. Dairy Technol., 29, 198-203.

[9] PARK (H. S.), MARTH (E. H.), Olson (N. F.) (1973). - Fate of enteropathogenic strains of Escherichia coli during the manufacture and ripening of Camembert cheese. J. Milk Food Technol., 36, 543-546.

[10] Dagnelie (P.) (1973). - Théorie et méthodes statistiques. Presses agronomiques, Gembloux, vol. 1, p. 92.

[11] EdWARDS (P. R.) and Ewing (W. H.) (1972). - Identification of Enterobacteriaceae. 3th Edition, Burgess Publishing Co., Minneapolis.

[12] Richard (C.) et Alonso (J. M.) (1976). - Une entérobactérie méconnue. Bull. Inst. Pasteur, 74, 339-352.

[13] Fantasia (L. D.), Mestrandrea (L.), Schrade (J. P.) and Yager (J.) (1975). Detection and growth of enteropathogenic Escherichia coli in soft ripened cheese. Appl. Microbiol., 29, 179-185.

[14] Mehlman (I. J.), Simon (N. T.), Sanders (A. C.) and Olson (J. C) Jr. (1974). Problems in the recovery and identification of enteropathogenic Escherichia coli from food. J. Milk Food Technol., 37, 350-355.

[15] Mrhlman (I. J.) Sanders (A. C.), Simon (N. T.) and Olson (J. C.) Jr. (1974). Methodology for recovery and identification of enteropathogenic Escherichia coli. J. Ass. Off. Anatyst. Chem., 57, 101-110.

[16] Fédération Internationale de Laiterie (1974). - Lait et produits laitiers. Dénombrement des bactéries coliformes. Norme Intern. FIL-IDF 73. 